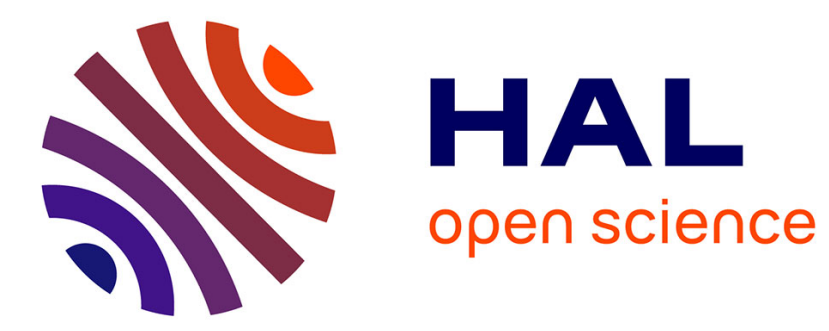

\title{
Aeronautical Ad Hoc Network for Civil Aviation
}

Quentin Vey, Alain Pirovano, José Radzik, Fabien Garcia

\section{To cite this version:}

Quentin Vey, Alain Pirovano, José Radzik, Fabien Garcia. Aeronautical Ad Hoc Network for Civil Aviation. Nets4 2014, 6th International Workshop, Nets4Cars/Nets4Trains/Nets4Aircraft 2014, May 2014, Offenburg, Germany. pp 81-93, 10.1007/978-3-319-06644-8_8 . hal-00989604

\section{HAL Id: hal-00989604 \\ https://hal-enac.archives-ouvertes.fr/hal-00989604}

Submitted on 20 May 2014

HAL is a multi-disciplinary open access archive for the deposit and dissemination of scientific research documents, whether they are published or not. The documents may come from teaching and research institutions in France or abroad, or from public or private research centers.
L'archive ouverte pluridisciplinaire HAL, est destinée au dépôt et à la diffusion de documents scientifiques de niveau recherche, publiés ou non, émanant des établissements d'enseignement et de recherche français ou étrangers, des laboratoires publics ou privés. 


\title{
Aeronautical Ad Hoc Network for Civil Aviation
}

\author{
Quentin Vey ${ }^{1}$, Alain Pirovano ${ }^{1}$, José Radzik $^{2}$, Fabien Garcia $^{1}$ \\ 1 ENAC, 7 avenue Édouard Belin, CS 5400531055 Toulouse cedex 4, France \\ 2 Université de Toulouse/ISAE, 10 avenue Édouard Belin, BP 54032, 31055 Toulouse \\ Cedex 4
}

\begin{abstract}
Aeronautical communication systems are constantly evolving in order to handle the always increasing flow of data generated by civil aviation. In this article we first present communication systems currently used for en-route aircraft. We then propose Aeronautical Ad hoc NETwork (AANET) as a complementary communication system and demonstrate its connectivity and assess the throughput by simulations based on real aircraft trajectories over the French sky and over the Atlantic ocean.
\end{abstract}

The final publication is available at link.springer.com :

http://link.springer.com/chapter/10.1007/978-3-319-06644-8_8

\section{Introduction}

Since the beginning of civil aviation, communications have always relied on direct link communication. As the traffic and the needs for safety grew, new technologies have been developed and implemented.

The airline traffic is expected to grow continuously in the coming years. This, combined with the migration from analog to digital and the new applications should lead to a saturation of the current communication systems capacity by 2020 [1].

The majority of data traffic in civil aviation application is observed between one aircraft and the ground. That's why new air-ground communication systems are expected. In this article, we propose AANET (Aeronautical Ad hoc Network) as an in-flight communication system to allow aircraft to communicate with the ground, in complement to other existing or future in-flight communication systems.

This paper is organized as follow: in section 2 we present some specificities of civil aviation communications, in section 3 the currently available communication systems are described as well as our proposal. This proposal is evaluated in section 4 and in section 5 our current research axes are given.

\section{Civil Aviation Communication Specificities}

In this section we present some properties and specificities of civil aviation communications. 


\subsection{Airspace Properties}

The upper airspace is almost exclusively used by commercial aircraft in cruise flight ("en-route" traffic). In the context of our study, we don't take into account the regulatory differences between airspaces in this section but focuses instead on the mobility patterns. We consider the two following zones:

- Continental: The continental traffic is generally characterized by numerous different routes, and a high aircraft density (especially above western Europe).

- Oceanic: As shown in Fig. 1, in oceanic area (and in remote continental area) the aircraft trajectories follow a limited set predefined routes, and aircraft density is low compared to continental airspace. In this case the aircraft traffic is similar to the cars traffic on a highway: the vehicles follow almost the same path, with similar speeds.

We consider here the particular case of the North Atlantic Tracks (NAT). These tracks define the routes followed by aircraft flying between Europe and north America. They are updated every day according to weather conditions in order to minimize fuel consumption and flight duration.

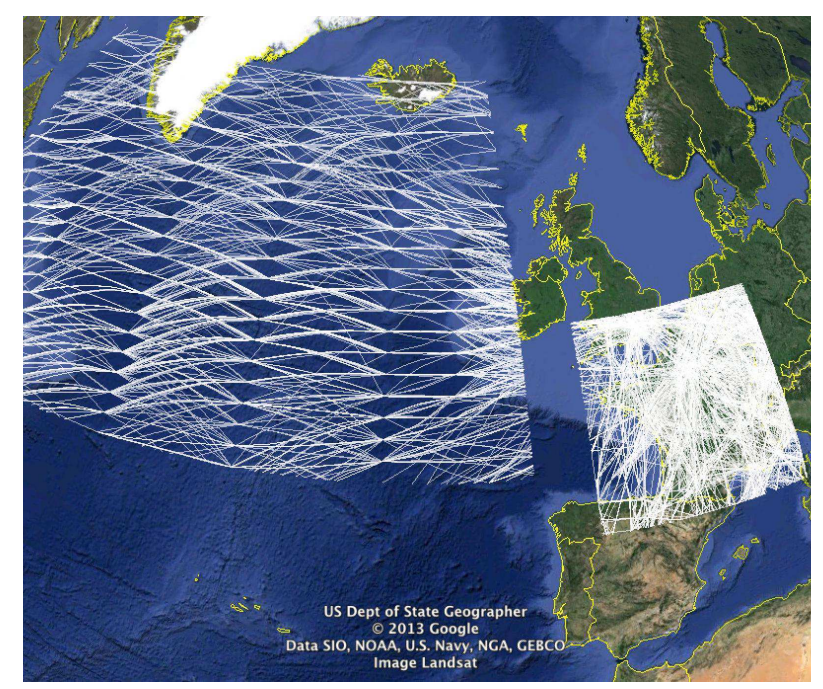

Fig. 1. Tracks of oceanic and continental traffic over $24 \mathrm{~h}$.

\subsection{Civil Aviation Communication Classification}

Four categories of communications are defined in the annex 10 of the International Civil Aviation Organization (ICAO) 2] regarding their safety level. 
- ATSC: Air Traffic Services Communication (critical). This class regroups communication between pilot and ATC to ensure the safety, speed and efficiency of the flight.

- AOC: Aeronautical Operation Control (critical). "Communication required for the exercise of authority over the initiation, continuation, diversion or termination of flight for safety, regularity and efficiency reasons" 2. Used by airline companies to communicate with aircraft (e.g. maintenance messages, fuel levels, exact departure and arrival time...).

- AAC: Aeronautical Administrative Control (non critical). "Communications necessary for the exchange of aeronautical administrative messages" 2 . AAC are neither linked to the security nor the efficiency of the flight. A few examples of AAC are informations regarding passengers (list of passengers, connections), special cleaning requests, hotel booking for flight attendants....

- APC: Aeronautical Passenger Communication (non critical). Examples of such communication are VoIP, e-mail, web browsing.

It has to be noted that critical communications follow very stringent international rules defined by ICAO (for example only some dedicated frequency band can be used) and are based on dedicated systems. These latter must meet very specific QoS requirements (transaction time, continuity, availability, integrity). These regulatory constraints does not apply to non critical communications, even if they may have to meet some requirements according to the applications (e.g. delay for passenger VoIP).

The current solution in civil aviation communication to ensure segregation is a physical segregation between critical communications and non-critical communications. The equipments aboard aircraft are physically different.

We will focus only on systems dedicated to critical services (ATSC, AOC) in the rest of this paper.

\section{Proposal of a Communication System}

\subsection{Civil Aviation Data Link Communication Systems}

Data link communication are used since the early 80 's. We list here some technologies currently used by civil aviation to communicate with en-route aircraft, and some technologies foreseen to be deployed in a near future.

Cellular Systems. Cellular systems provide a direct link between the aircraft and a ground station. With the exception of the HFDL, all these cellular systems are limited to a line-of-sight (or lower) range, thus requiring the deployment of a large ground infrastructure to cover a region and unable to cover oceanic flights far from the shores. The offered capacity is shared between all aircraft within range of ground station. Moreover, for VDL there is a need for handover procedure generally based on ground comunication sytem between stations. Despite these drawbacks, they offer a reliable service at lower cost than satellitebased systems, and are suitable for numerous continental flights. 
Existing and Future Cellular Systems.

- HFDL: High Frequency Data Link. Standardized in 1999, it offers a link capacity up to $1.8 \mathrm{kbit} / \mathrm{s}[3$. (shared between every aircraft within range of a station). Because of the unique propagation of the HF radio a single station can cover a very large area (range is usually around $2500 \mathrm{~km}$, can go up to $4000 \mathrm{~km}$ depending on ionospheric conditions). This very long range enables a global coverage (including polar areas) using only 14 stations. The major drawback of this system is its low capacity.

- VDL mode 2: VHF Data Link. Specified in 1996 by the ICAO, it offers a $31.5 \mathrm{kbit} / \mathrm{s}$ capacity $[3$ and a line-of-sight coverage $(400 \mathrm{~km})$.

- L-DACS: L-DACS is foreseen by the Single European Sky ATM Research (SESAR) project as a new data link communication systems, expected in 2016. It should operate in L-band and offer a 200 kpbs capacity.

Satellite-based Systems. Satellite systems offer a link between two transceivers (an aircraft and a control center for example) by using satellites as relay. A satellite-based systems provides wide coverage and relatively high speed links, but at the expense of a high cost. Main types of satellites are:

- GEO: Geostationary Earth Orbit. A satellite on GEO appears on a fixed position in the sky and covers one third of the Earth. The drawback of this position are high delays due to the distance, and a lack of coverage of the polar regions. It has also to be noted that the integration of a high-gain antenna (required for high-speed connections) on an aircraft is a complex and costly task.

- LEO: Low Earth Orbit. A satellite on LEO will have a lower delay and can cover polar regions, but will appear moving in the sky, thus requiring a whole constellation to enable a continuous coverage.

Existing and Future Satellite-based Systems.

- Inmarsat: InmarSat is a GEO satellite operator and a communication service provider, providing connection from $1.2 \mathrm{kbit} / \mathrm{s}$ to $10.5 \mathrm{kbit} / \mathrm{s}$ for critical services (Aero L and Aero $\mathrm{H} / \mathrm{H}^{+}$products).

- Iridium: Iridium provides communications using LEO satellites, offering 2.4 $\mathrm{kbit} / \mathrm{s}$ links.

- IRIS: SESAR project has defined an GEO satellite solution in partnership with the European Space Agency (ESA) called IRIS, the operational solution is expected in 2020. The offered capacity should be several dozens of kbit/s.

Conclusion on Existing and Future Communication Systems. Table 1 summarizes the main properties of these systems currently used or in development for critical applications in civil aviation. However the traffic growth and the new expected applications should saturate these current communication systems by 2020 [1] 
We would like to underline the offered capacity and covered areas. These values are indeed quite unsual for most ground network where high capacity means often several Mbps, and must be kept in mind when studying the performances of communication systems designed for civil aviation critical communications.

In this context, communications during oceanic flights (or over remote continental areas) implies that the aircraft is equipped with at least the only two currently available communication systems for critical communications, which are the more often for this area HFDL and satellite link. Furthermore above polar regions (latitude higher than $70^{\circ}$ ) the only satellite system available is Iridium.

Table 1. Summary of the performances of several communication systems for civil aviation

\begin{tabular}{|c|c|c|c|}
\hline System & Offered capacity & range / coverage & currently operational \\
\hline HFDL & $\begin{array}{c}1.8 \mathrm{kbit} / \mathrm{s} \\
\text { per ground station }\end{array}$ & $2500 \mathrm{~km}$ & yes \\
\hline VDL mode 2 & $\begin{array}{c}31.5 \mathrm{kbit} / \mathrm{s} \\
\text { per ground station }\end{array}$ & $400 \mathrm{~km}$ & yes \\
\hline L-DACS & $\begin{array}{c}200 \mathrm{kbit} / \mathrm{s} \\
\text { per ground station }\end{array}$ & $400 \mathrm{~km}$ & no (under test) \\
\hline InmarSat aero L & $\begin{array}{c}1.2 \mathrm{kbit} / \mathrm{s} \\
\text { per aircraft }\end{array}$ & for lat. under $70^{\circ} \mathrm{N} / \mathrm{S}$ & yes \\
\hline InmarSat aero H/H $/ \mathrm{H}^{+}$ & $\begin{array}{c}10.5 \mathrm{kbit} / \mathrm{s} \\
\text { per aircraft }\end{array}$ & for lat. under $70^{\circ} \mathrm{N} / \mathrm{S}$ & yes \\
\hline Iridium & $\begin{array}{c}2.4 \mathrm{kbit} / \mathrm{s} \\
\text { per aircraft }\end{array}$ & global & yes \\
\hline IRIS & $\begin{array}{c}\text { dozens of kbit/s } \\
\text { per aircraft }\end{array}$ & for lat. under $70^{\circ} \mathrm{N} / \mathrm{S}$ & no (expected 2020) \\
\hline
\end{tabular}

\subsection{AANET as a Civil Aviation Communication System}

In this context, particularly in oceanic airspace, we propose AANET as a new complementary solution to send messages from the aircraft to the ground. The main expected advantage is that, an aircraft could send/receive a message to the ground even if no ground station is in line of sight.

Others expected advantages at this step of the study are the following:

- Simple and relatively light ground infrastructure required,

- Simple and relatively light embedded system,

- Redundancy of the path in the network particularly when aircraft density is high (multipath). 
- Load-balancing ability when several path are available.

Nevertheless AANETs present several issues. For instance, the connectivity relies on aircraft density in the considered airspace and the direction from aircraft to the ground will result in a tree logical topology in which ground gateways or on some parts of the network may represent bottlenecks.

\subsection{Proposed Architecture for Access Layers}

The AANET architecture we propose implements omnidirectional radio links between the different nodes with two types of links: a2a (aircraft to aircraft) and a2i (aircraft to infrastructure or ground stations), with a bandwidth of $20 \mathrm{MHz}$ in the $2 \mathrm{GHz}$ band (actual frequency will depend on radio spectrum availability). To manage concurrent accesses to the media three allocation methods have been considered: by frequency (Frequency Division Multiple Access, FDMA), by time (Time Division Multiple Access, TDMA) and by spreading code (Code Division Multiple Access, CDMA). Combinations of these methods are possible. However, frequency division cannot share the available bandwidth in the considered topology without significantly reducing the available bandwidth for every connection. And time division raises an important issue on clock synchronization. Therefore, we propose the use of Direct Sequence CDMA (DS-CDMA), a solution also used in 3G mobile, networks and proposed in IRIS [4. DS-CDMA has the major advantage that it does not require any coordination between nodes and it allows multiple simultaneous transmissions. The collisions which occur can be resolved in the receiver thank to the low intercorrelation between two different and well chosen spreading codes. It has to be noted that this collision recovery can only be done within the limits of Multiple Access Interferences (MAI). This represents the major limiting factor of the performance of CDMA systems. This particular point has been investigated in [5]

\section{Feasibility and Performance Assessment}

\subsection{Connectivity Assessment}

Related Work. Previous studies on AANET, for example the ATENAA [6] project and NewSky project [1] and [7, have studied the feasibility of an ad-hoc network. 1] and 7] are focused on the connectivity of airplanes over the North Atlantic Tracks by assuming that the aircraft took off and landed at scheduled time and followed the shortest path (geodesic trajectory) between departure and arrival airport. They conclude to the feasibility of a such network. However, as shown in Fig. 2, differences as high as $1000 \mathrm{~km}$ are observed between real trajectory and shortest path. Also, the eastbound and westbound traffic do not follow the same tracks at all: aircraft flying from Europe to America tend to follow a route going several hundreds kilometers north of the shortest path in order to avoid the jet-stream, a high-altitude powerful wind blowing eastward. 


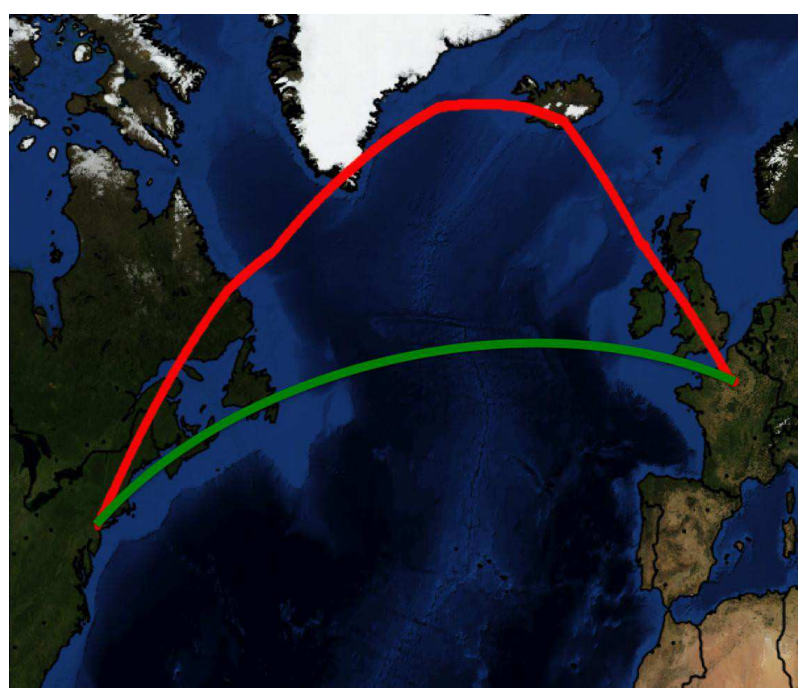

Fig. 2. Difference between the shortest path trajectory (green) and the real trajectory (red) for a Paris-New York flight on the $16^{\text {th }}$ of September 2011.

Methodology. In order to avoid this bias, our study is based on the replay of real traffic traces from the French civil aviation authority and Eurocontrol based on either radar data or position report from the aircraft itself. If a time-scale smaller than the one provided by the reports is required, intermediate positions are interpolated between these reported positions. In the rest of this paper, we will refer to these as "pseudo-real" positions.

Because of these datasets, our study focus on the north Atlantic (for oceanic traffic) and French (for continental traffic) airspaces. For the continental study, the ground stations have been placed near air traffic control centers, and for oceanic airspace they have been positioned on the coasts and islands along the tracks.

Connectivity has been assessed with a software we have specifically developed, AeRAN (for Aeronautical Ad hoc Networks). It processes the datasets presented in the previous paragraph and a list of ground stations, then gives several statistics as output such as the amount of aircraft connected to the ground, the ratio of aircraft connected directly or via several aircraft to a ground station. In this part, because only the connectivity is being evaluated, computations are made under the assumption that links are ideal, i.e. it is possible to send data to any aircraft within a given range without interference.

Results. The software presented in the previous part has been used to compute the number of aircraft connected to a ground station (directly or through a multi- 
hop path) in function of the time of day for several link ranges (100, 200 and $400 \mathrm{~km})$. The results are presented in Fig. 3.
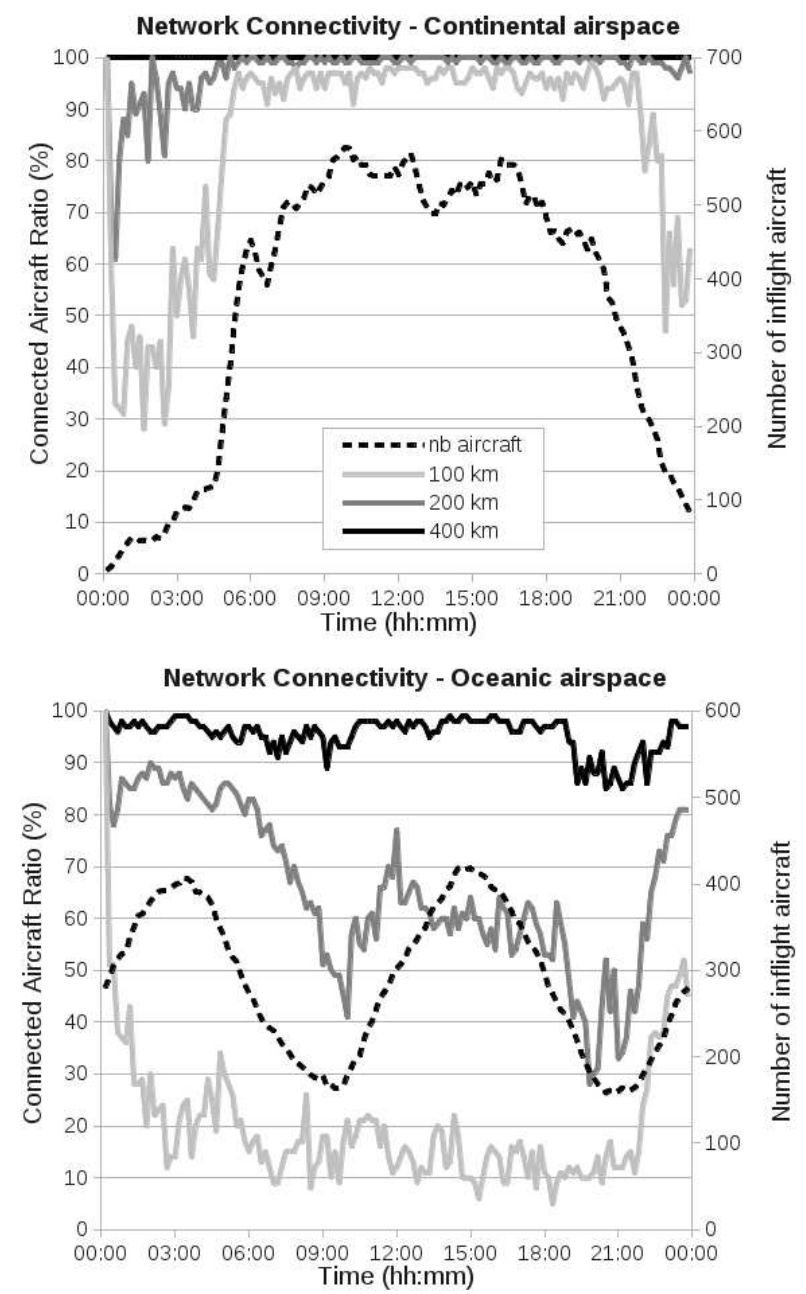

Fig. 3. Communication range influence on network connectivity.

These results underline the influence of the communication range on the aeronautical ad hoc network connectivity (solid lines and left y axis) in the considered continental airspace and oceanic airspace. As expected, the connected aircraft ratio increases with the communication range up to a point. And after this point increasing communication range does not have significant impact on 
connectivity. These figures, on which the number of instantaneous flying aircraft has been displayed (dotted lines and right y axis), let also think that the this number and connectivity are correlated. We can see here that in the French sky a communication range of $150 \mathrm{~km}$ allows a mean connectivity of $90 \%$ of the aircraft during the day, with $99 \%$ of aircraft connected between 6:00 and 21:00. But in the oceanic airspace, this communication range should be $350 \mathrm{~km}$ in order to ensure a mean connectivity greater than $90 \%$ in the day. These latter results are explained by the fact that the aircraft density is lower in the oceanic airspace. It is important here to note again that aeronautical ad hoc networks have to be considered more as an additional mean for air-ground communication than as an alternative stand-alone one. Hence, these results let us foresee the benefits of this solution particularly considering its low cost relatively to other solutions.

\subsection{Throughput Assessment}

Methodology. TCP propose several functionalities and mechanisms to fairly adapt its sending rate to the network available capacity. So we use this transport protocol in a "reno-like" version in order to assess the effective mean offered capacity to each aircraft in the AANET . We have developed a simulation model for aeronautical ad hoc network with OPNET Modeler (Riverbed), which is a discrete event simulator for all kind of networks. The aim of the model is to simulate aeronautical ad hoc network in a realistic way. Aircraft positions are defined using the same method and inputs data as in our homemade tool AeRAN as explained in section 4.1. Considering physical layer properties the capacity of each inter nodes link is set to $1 \mathrm{Mbit} / \mathrm{s}$.

To determine the available throughput per aircraft, a path to the ground station has to be chosen for each of them. At this step, we use the shortest path, regarding the distance between the nodes, to the closest ground station given by the Dijkstra algorithm. Thus we obtain a list of edges representing the path from each aircraft to the ground station. The maximum distance to establish a connection between two aircraft (their communication range) is a simulation parameter. We used the link ranges that produced a mean connectivity of $90 \%$ found in the previous part (350 km for oceanic, $150 \mathrm{~km}$ for continental). We also made the hypothesis that there were enough spreading codes available for every aircraft. However, link interferences for simultaneous transmissions are taken into account in this part.

At each time of the simulation, we consider a graph whose nodes are aircraft and ground stations, and whose edges are the available connections between them. We assume that a connection is always available between all pairs of ground stations (based on a ground network infrastructure). The weight of each edge in the graph is its length. Then, the Dijkstra algorithm is used to find in this graph the shortest path from each aircraft to a ground station. It gives for each connected aircraft a path to the closest ground station. Finally, considering the application level, as the idea is to assess the available throughput for each

aircraft, we use greedy TCP sources in our model. Such TCP sources generate new TCP segments as soon as the previously sent ones are acknowledged. Hence, 
data are generated at the highest possible rate regarding the aeronautical ad hoc network congestion and the obtained results indicates the expected throughput that could be achieved by each aircraft.

Results. Table 2 shows the mains results obtained after a simulation campaign. The

Table 2. Shortest path routing results

\begin{tabular}{|c|c|c|c|}
\hline & $\begin{array}{c}\text { Mean aircraft throughput } \\
\mathrm{kbit} / \mathrm{s}\end{array}$ & $\begin{array}{c}\text { Max delay (95\% pkts) } \\
\mathrm{ms}\end{array}$ & $\begin{array}{c}\text { Mean delay } \\
\mathrm{ms}\end{array}$ \\
\hline Continental & 38,3 & 551 & 401 \\
\hline Oceanic & 68,2 & 426 & 184 \\
\hline
\end{tabular}

These quite better throughput performances in oceanic airspace are mainly explained by the fact that, in the considered oceanic airspace, the ratio between the number of ground stations and the number of aircraft is better. Actually there are a maximum of about 400 simultaneous flying aircraft (with a mean of 275 aircraft) for 8 ground stations during one day. Comparatively, in the French sky, our case study for continental area, the PIAC (Peak Instantaneous Aircraft Count) is near 600 aircraft with a mean of 500 simultaneous flying aircraft between 6:00 and 21:00 for 5 ground stations.

Hence, we can see that, in this worst-case scenario (TCP greedy) with a very simple routing algorithm and a basic Reno-like TCP congestion control algorithm, AANET performs better than any currently operational system for critical communications in terms of throughput per aircraft.

\subsection{Assessment with a Realistic Application}

As an example of realistic application, we consider one proposed by the BEA (in English: Bureau of Inquiry and Analysis for Civil Aviation Safety) which is the French authority responsible for safety investigations into accidents or incidents in civil aviation. The main objective of this application is to make easier data recovery from flight recorders by periodically sending flight parameters to the ground in oceanic airspace. Three sets of parameters which should be sent each second for a given aircraft have been defined 8 . The bulkier set has a size of 12,288 bits. So we created a specific model in a new scenario which allows each aircraft to behave as if it sends a set of flight data of 12,288 bits each second. Of course this type of traffic source exhibits higher burstiness than the one used in the previous sections. The simulation results show that all packets reach successfully the ground station. The maximum observed delay for $95 \%$ of packet is $178 \mathrm{~ms}$. The proposed innovative communication system is therefore compatible with such a realistic application. 


\section{Further Work}

\subsection{Disruption Tolerance}

Regarding the speed of airliners, the topology of AANET is highly dynamic, particularly in the considered continental airspace. A single aircraft or a subset may be temporarily disconnected from the rest of the AANET. Some little disruptions may not be considered as full disconnection, depending on the maximum tolerated end to end delay for our applications. For a given link range, a disruption tolerance should increase connectivity, and for a given connectivity, these tolerated disruptions should allow to lower the transmission power, thus reducing interferences and increase throughput.

The dynamicity of the network topology is currently being assessed with the software presented in 4.1 and the same "pseudo-real" trajectories in order to evaluate the improvement offered by the tolerated disruptions. Depending on the results of this study, Disruption/Delay Tolerant Network (DTN) 9]) ability will be considered to improve connectivity. The DTN paradigm makes a network robust to high delays or disruptions by taking advantage of the movement of the nodes to improve connectivity.

\subsection{CDMA Code Allocation}

In the throughput study we assumed that there were enough spreading codes of a given length for every aircraft and that a given code was statically assigned to each aircraft, which is a simplification of our problem. In reality the spreading code attribution algorithm should be adaptative, distributed, enable spatial reuse of codes and give the shortest possible code for every link in order to maximize link throughput. The codes themselves also must allow this. Orthogonal Variable Spreading Factor (OVFS) are a family of spreading codes that meets these requirement by providing orthogonal spreading codes of different length, i.e. codes of different length which enable concurrent transmission.

Jiang et Al. proposed in [10] an interesting code attribution algorithm. It is based on a RTS/CTS mechanism with spreading code negotiation on a signaling channel, then a data transfer with the negociated code. During the negotiation, each node evaluates the required length of the spreading code based on the network activity in his neighborhood. It has been tested with simulated aircraft movement and must now be tested with our "pseudo-real" aircraft traffic data. We are also working on some improvement of this algorithm that have to be implemented and assessed.

\subsection{Investigation of Different Routing Algorithms}

We used in this paper a very simple routing algorithm (shortest geographic distance). In the rest of our research, we plan to investigate routing algorithms based more advanced metrics. For example, the following metrics seems interesting to choose the routing path: 
- Number of relays: minimizing the number of relays should reduce the total processing delay and the collision.

- Link durability: a higher link durability could also be preferred in order to reduce the amount of disconnections. In this case, link stability can be evaluated with Doppler frequencies: a lower Doppler frequency between two aircraft means that their trajectories (speed and heading) are similar and that the link is less likely to be interrupted.

- Network load: because an AANET will offer several different routes (depending on aircraft density), the routing algorithm could perform load balancing by choosing routes according to the load of the relay nodes.

Of course, combinations of these metrics and others are possible.

Clustering is also another solution that could enhance the AANET performances. To build clusters one can use the same metrics as the one presented above, for example [1] uses similarities in the trajectories in order to create long-lasting clusters.

Several routing algorithms from the MANET and the VANET communities uses these metrics and should be considered for AANETs (similarities between VANET and MANET have been studied in [12, e.g. TOPO [13, DSR [14], GPSR [15], MUDOR [16. AANET specific routing algorithm have also been proposed, e.g. ARPAM[16], GLSR[17], AeroRP 18].

Unfortunately, performances of these routing algorithms are rarely assessed with real aircraft trajectories. In our further work we will test the most interesting ones with simulations based on the "pseudo-real" trajectories presented in 4.1 .

\section{Conclusion}

Considering traffic growth, new expected applications and the limitation of existing systems, new communication systems will be needed for civil aviation in the future, especially for oceanic flights. In this article we proposed AANET as a complement to the cellular and satellite communication systems. Our study demonstrates that its throughput performance are sufficient to cope with current and some new applications. However its connectivity is heavily dependent on the aircraft density. But, because it performs better when aircraft density is high, AANET can relieve other communication systems that would otherwise be overloaded under these conditions.

We end this paper with some research axes that will be investigated in our further works in order to improve the overall performances of air ground communication based on AANET.

\section{References}

1. M. Schnell and S. Scalise. Newsky - concept for networking the sky for civil aeronautical communications. Aerospace and Electronic Systems Magazine, IEEE, 22(5):25-29, May 2007. 
2. International Civil Aviation Organisation. ICAO annex 10, November 2005.

3. ICAO annex 10 vol. 3, July 2007.

4. Indra. Antares communication standard design definition file. Technical note IRISAN-CP-TNO-610-ESA-C1, ESA, September 2013. DRL Nr: D020.

5. F. Besse, A. Pirovano, F. Garcia, and J. Radzik. Interference estimation in an aeronautical ad hoc network. In Digital Avionics Systems Conference (DASC), 2011 IEEE/AIAA 30th, pages 4C6-1-4C6-11, Oct 2011.

6. Massimiliano Amirfeiz. ATENAA project : Advanced technologies for networking in aeronautical applications. In aerodays 2006, Vienna, June 2006.

7. D. Medina, F. Hoffmann, S. Ayaz, and C.-H. Rokitansky. Feasibility of an aeronautical mobile ad hoc network over the north atlantic corridor. In Sensor, Mesh and Ad Hoc Communications and Networks, 2008. SECON '08. 5th Annual IEEE Communications Society Conference on, pages 109-116, June 2008.

8. BEA. transmission declenchee de donnees de vol (triggered flight data transmission). Technical report, Bureau d'Enquètes et d'Analyses pour la sécurité de l'aviation civile, March 2011.

9. S. Ali, J. Qadir, and A. Baig. Routing protocols in delay tolerant networks - a survey. In Emerging Technologies (ICET), 2010 6th International Conference on, pages $70-75$, Oct 2010 .

10. Zaihan Jiang and Mengchu Zhou. Spread spectrum MAC protocol with dynamic rate and collision avoidance for mobile ad hoc network. IEEE Transactions on Vehicular Technology, 56(5):3149-3158, September 2007.

11. Wei Fan, Yan Shi, Shanzhi Chen, and Longhao Zou. A mobility metrics based dynamic clustering algorithm for VANETs. In IET International Conference on Communication Technology and Application (ICCTA 2011), pages 752-756, 2011.

12. Mickaël Royer, Alain Pirovano, and Fabien Garcia. Survey on context-aware Publish/Subscribe systems for VANET. In Marion Berbineau, Magnus Jonsson, JeanMarie Bonnin, Soumaya Cherkaoui, Marina Aguado, Cristina Rico-Garcia, Hassan Ghannoum, Rashid Mehmood, and Alexey Vinel, editors, Communication Technologies for Vehicles, number 7865 in Lecture Notes in Computer Science, pages 46-58. Springer Berlin Heidelberg, January 2013.

13. Wenjing Wang, Fei Xie, and M. Chatterjee. An integrated study on mobility models and scalable routing protocols in VANETs. In 2007 Mobile Networking for Vehicular Environments, pages 97-102, 2007.

14. D. Johnson, Y. Hu, and D. Maltz. rfc4728 : Dynamic source routing, February 2007.

15. Brad Karp and H. T. Kung. Gpsr: Greedy perimeter stateless routing for wireless networks. In Proceedings of the 6th Annual International Conference on Mobile Computing and Networking, MobiCom '00, pages 243-254, New York, NY, USA, 2000. ACM.

16. K. Karras, T. Kyritsis, M. Amirfeiz, and S. Baiotti. Aeronautical mobile ad hoc networks. In Wireless Conference, 2008. EW 2008. 14th European, pages 1-6, June 2008.

17. D. Medina, F. Hoffmann, F. Rossetto, and C.-H. Rokitansky. A geographic routing strategy for north atlantic in-flight internet access via airborne mesh networking. Networking, IEEE/ACM Transactions on, 20(4):1231-1244, Aug 2012.

18. Kevin Peters, Abdul Jabbar, Egemen K. Cetinkaya, and James PG Sterbenz. A geographical routing protocol for highly-dynamic aeronautical networks. In Wireless Communications and Networking Conference (WCNC), 2011 IEEE, pages 492497, 2011. 